Weichenstellung zur Nachhaltigkeitsberichterstattung mit Zukunft

\section{Die dritte Generation der Leitlinien der Global Reporting Initiative}

\author{
Seit rund vier Jahren werden die „Sustainability Reporting \\ Guidelines" angewandt. Nach einem umfassenden Überarbei- \\ tungsprozesses veröffentlicht die Global Reporting Initiative im \\ Oktober 2006 die dritte Generation des Leitfadens \\ zur Nachhaltigkeitsberichterstattung (G3). Für Berichterstatter \\ und Berichtsnutzer bietet der $\mathrm{G} 3$ interessante Neuerungen. \\ Von Ralf Isenmann
}

D e Nachhaltigkeitsberichterstattung kennzeichnet einen neuen ganzheitlichen Ansatz der Unternehmenspublizität. Sie verbindet drei bislang isolierte Berichterstattungen miteinander und fügt deren Interdependenzen in einer integrativen Betrachtung zusammen. Die Bezeichnungen der thematisch umfassenden Nachhaltigkeitsberichte variieren beträchtlich. Unabhängig von Begriffsvielfalt und konzeptionellen Entwicklungslinien hinter den einzelnen Bezeichnungen ist für diese Berichterstattung eine integrative Darstellung charakteristisch. Und zwar zumeist anhand der drei Bereiche Ökonomie, Ökologie und Soziales.

\section{Bestrebungen zur Standardisierung}

Ebenso vielfältig wie die Interessen an der Nachhaltigkeitsberichterstattung, so verschieden erscheinen auch die Vorgaben, mit denen eine Standardisierung angestrebt wird. „The Corporate Responsibility Codebook“ enthält allein 32 einschlägige Vorgaben zu diesem Bereich. Eine Sonderstellung bei den standardsetzenden Akteuren nimmt die Global Reporting Initiative (GRI) ein. Aus institutioneller Sicht repräsentiert sie eine internationale Multi-Stakeholder-Organisation. Aus inhaltlicher Sicht haben sich die GRI-Leitlinien mittlerweile als De-facto-Standard herausgebildet.

Die dynamische Entwicklung in der Nachhaltigkeitsberichterstattung, verbun- den mit der Vielzahl unterschiedlicher Vorgaben, macht die Nachhaltigkeitsberichterstattung insgesamt unübersichtlich. Neben der Beachtung exogener Bestimmungsfaktoren sind Unternehmen vor interne Herausforderungen gestellt, indem sie die zu vermittelnden Themen inhaltlich stimmig zusammenzustellen sowie ein effizientes und arbeitsteiliges Informationsmanagement quer zu traditionellen Funktionsbereichen zu organisieren haben. Für Berichterstatter ist Nachhaltigkeit ein offenkundig schwieriges Feld. Den Berichtsnutzern erschwert dies Leistungsvergleiche, Unternehmensanalysen, Benchmarks, Ratings und Rankings.

Allerdings steigen die Anzeichen einer konsequenteren Standardisierung, vor allem seit professionelle Berichtsnutzer in der Finanzwelt begonnen haben, ökologische und soziale Leistungsindikatoren in ihre Bewertungen mit einzubeziehen. Insofern erscheinen Nachhaltigrelevant und werden deshalb auch zum Thema der obersten Unternehmensführung. Gerade das Interesse professioneller Berichtsnutzer fördert eine Konvergenz zu harmonisierten Dokumentstrukturen und einem standardisierten Datenformat mit semantischer Auszeichnung. Mit dessen Hilfe können Inhalte aus Nachhaltigkeitsberichten ohne nochmalige manuelle Eingabe einfach ausgetauscht und automatisiert ausgewertet werden. Der Einsatz moderner Informations- und Kommunikationstechnologien keitsberichte zunehmend wettbewerbs-
(IKT) und Methoden im Informationsmanagement begünstigt solche Vereinheitlichungen.

\section{Revision von Leitlinien und Rahmenwerk}

Das Ziel der GRI besteht darin, weltweit akzeptierte Leitlinien zur Nachhaltigkeitsberichterstattung zu entwickeln und so insgesamt deren Qualität, Aussagekraft und Verbreitung zu steigern.

Nach zweijähriger intensiver Revision folgten im August 2002 die zweiten der ersten Leitlinien „Sustainability Reporting Guidelines" (Generation der GRI 2002). Wiederum vier Jahre später münden die gesammelten Erfahrungen sowie die Erkenntnisse eines umfassenden Überarbeitungsprozesses in die dritte Generation des Leitfadens, der im Oktober 2006 veröffentlicht wird.

Kennzeichnend für die jeweilige Revision bei GRI ist ein „Structured Feedback Process“, der Interessierten zahlreiche Möglichkeiten zur kritischen Mitarbeit bietet. Instrumente der Mitarbeit sind unter anderem Online-Befragungen, Kommentierungsphasen sowie spezifische Arbeitsgruppen und Runde Tische. Neben dem Spektrum an Partizipationsformen ist es ein Anliegen der GRI, ein möglichst breites Feld unterschiedlicher Akteure in die Weiterentwicklung der Leitlinien einzubeziehen, institutionell wie auch regional. So werden Unternehmensvertreter, gemeinnützige Organisationen, berufsständische Experten wie Wirtschaftsprüfer, Unternehmensberater und Kommunikationsspezialisten, Investorengruppen, Gewerkschaften, Wissenschaftler sowie Vertreter aus Rating- und Rankingorganisationen einbezogen.

Um das Kernstück, das die eigentlichen Leitlinien bilden, hat die GRI seit 2002 eine Reihe ergänzender Dokumente entwickelt. Technische Protokolle (Technical Protocols) spezifizieren die Messung von Indikatoren wie zum Beispiel Energie. Branchenergänzungen (Sector Supplements) widmen sich wirtschaftszweigspezifischen Besonderheiten, beispielsweise für die Automobilbranche. Themenspezifische Leitfäden (Issue Guidance Docu- 
ments) helfen bei der Interpretation erklärungsbedürftiger Themen wie etwa Diversität. Zusammen mit den Leitlinien bilden technische Protokolle, Branchenergänzungen und themenspezifische Leitfäden als „Family of Documents“ ein umfangreiches Rahmenwerk zur Nachhaltigkeitsberichterstattung. Hinzugekommen sind neuerdings „Linkage Papers“, die die Verbindungen der GRI-Leitlinien zu anderen Vorgaben wie etwa zum Global Compact verdeutlichen.

\section{Neuerungen durch G3}

Die Neuerungen, die G3 im Vergleich zu den GRI-Leitlinien 2002 (G2) mit sich bringen enthalten institutionelle, angebotsspezifische, formale und inhaltliche Aspekte:

- Institution: Die GRI überarbeitet ihr Geschäftsmodell. Weg von einem weithin philanthropisch geprägten Charakter eines Start-up, dessen Finanzierung sich weitgehend von Spenden und Mitgliedsbeiträgen speist, hin zu einer selbstfinanzierten, unabhängigen Institution. Diese Professionalisierung zielt auch auf die Entwicklung der GRI als maßgeblicher Akteur mit einer dauerhaften Sicherung ihres Einflusses als internationale Multi-Stakeholder-Institution mit standardsetzender Geltung für die Nachhaltigkeitsberichterstattung.

- Angebote: Als Folge des neuen Geschäftsmodells erweitert die GRI das Angebot um neue Produkte und Dienstleistungen. Darunter sind Informationsprodukte und -dienstleistungen wie der Relaunch der GRI-Website, die Dokumentstruktur eines Nachhaltigkeitsberichts gemäß eXtensible Business Reporting Language (XBRL) und die Report-Registrierung, ein differenzierter Service zu Schulung, Weiterbildung und Training zur Nachhaltigkeitsberichterstattung sowie GRIAkkreditierungsprogramme.

| Formalia: Der Umfang der „Sustainability Reporting Guidelines" hat sich im vorliegenden Entwurf erheblich verschlankt. Von ehemals 94 Seiten bei G2 bleiben nunmehr 27 Seiten bei
G3. Ferner hat die GRI die Gliederung der Leitlinien gestrafft. G3 besteht aus insgesamt fünf Abschnitten: (I) Überblick über die Nachhaltigkeitsberichterstattung (2 Seiten), (II) Berichtsinhalt, -grenzen und -qualität (7 Seiten), (III) Angaben eines Standardberichts (11 Seiten), (IV) Anwendung der Leitlinien (2 Seiten), (V) Glossar mit Begriffsdefinitionen (2 Seiten). Doch diese Anordnung folgt eher einem prozessorientierten Ablauf in der Nachhaltigkeitsberichterstattung. Ferner hat die GRI zur Vorbereitung auf G3 eine eigene Informations- und Kommunikationsplattform im Internet mit zahlreichen Hinweisen, Dokumenten und Hintergrundinformationen eingerichtet.

Inhalt: Aus der Fülle der Detailänderungen seien hier drei Punkte hervorgehoben): (I) Die umfassend eingeführten elf Einzelprinzipien der Nachhaltigkeitsberichterstattung aus G2 wurden in zwei Gruppen, „Issue Determination“ und „Quality of Data“, gebündelt. Sie wurden begrifflich verdichtet sowie durch Beispiele und Fragen veranschaulicht. (II) Die gestraffte Gesamtstruktur der G3 spiegelt sich in den empfohlenen Angaben eines Standardberichts wider. Demnach soll ein Standardbericht nach GRI zumindest drei Bereiche enthalten. Erstens Angaben zur Unternehmensstrategie, zum Organisationsprofil, zu Berichtsmerkmalen, zu Governance, Stakeholder-Beziehungen und Verpflichtungen (Disclosure Items). Zweitens Angaben zu Managementsystemen (Disclosure on Management Approach) sowie drittens Angaben zu den Leistungskennzahlen (Disclosure on Performance Indicators). (III) Die Leistungsindikatoren wurden begrifflich entschlackt und konzeptionell überarbeitet. Sie wurden auch im Hinblick auf bestehende internationale Standards sowie durch technische Protokolle spezifiziert. Die insgesamt 97 Einzelindikatoren bei G2 wurden auf 79 Indikatoren bei G3 reduziert. Unverändert blieben die fünf Bereiche, die der Bündelung der Indikatoren dienen. Diese sind Ökonomie, Umwelt, Menschenrechte, Arbeit sowie Produktverantwortung und Gesellschaft.

Insgesamt lassen sich die Neuerungen der G3 durch die Verschlankung ihres Umfangs, die Straffung ihrer Struktur sowie die präziseren Angaben zu Berichtsinhalten auch quantitativ nachvollziehen. Während ein Nachhaltigkeitsbericht mit vollständigem Inhalt ohne Branchenergänzungen nach G2 insgesamt 395 inhaltliche Bestandteile enthält, reduziert sich diese Anzahl bei G3 auf rund 260 Bestandteile, so die Ergebnisse einer semantischen Dokumentanalyse.

\section{Resümee und Ausblick}

Wie sich die Neuerungen der G3 letztlich auf die Entwicklung der Nachhaltigkeitsberichterstattung auswirken mögen, läßt sich im Vorfeld kaum treffsicher abschätzen. Ob sie tatsächlich einen quantititiven Schub auslösen, die Verbreitung der Nachhaltigkeitsberichterstattung wirksam befördern und insbesondere Unternehmen zu einer nachhaltigen Entwicklung ermuntern, hängt sicherlich auch vom Einsatz in der Betriebsroutine ab und dies muss insofern erst der Belastungstest in der Praxis zeigen.

Gleichwohl scheinen die Weichen in Richtung Zukunft gestellt. Dies gilt insbesonere im Blick auf die Anforderungen einer modernen (Online-) Nachhaltigkeitsberichterstattung, in der relevante Inhalte, Darstellungen in unterschiedlichen Medien, differenzierte Präsentationsformen (medienspezifisch, zielgruppenorientiert) und sich ergänzende Verteilungsmechansimen zu maßgeschneiderten Kommunikationsinstrumenten kombiniert werden können.

AUTOR + KONTAKT

Dr. Ralf Isenmann ist Dozent am Fachbereich Wirtschaftswissenschaft der Universität Bremen und Mitarbeiter am Institut für Projektmanagement und

Innovation (IPMI). Universität Bremen, PMI, Wilhelm-Herbst-Strasse 12, 28359 Bremen. Tel.: 0421/218 8202,

E-Mail: ralf.isenmann@innovation.uni-bremen.de 
(c) 20I0 Authors; licensee IÖW and oekom verlag. This is an article distributed under the terms of the Creative Commons Attribution Non-Commercial No Derivates License (http://creativecommons.org/licenses/by-nc-nd/3.o/), which permits unrestricted use, distribution, and reproduction in any medium, provided the original work is properly cited. 